\title{
A Novel Missense ZBTB18 Mutation Identified in an Intellectual Disability Family by Whole Genome Sequencing
}

Nana Li

Sichuan University

Lu Li

Sichuan University

Yanna Zou

Sichuan University

Zhen Liu

Sichuan University

Ying Deng

Sichuan University

Hong Qin

Sichuan University

Xiaoqiong Qiu

Sichuan University

Hong Kang

Sichuan University

Yanping Wang

Sichuan University

Jun Zhu

Sichuan University

Meixian Wang

Sichuan University

pingyu ( $\nabla$ yup@scu.edu.cn )

Sichuan University

\section{Research}

Keywords: intellectual disability, whole genome sequencing (WGS), ZBTB18, c.1323C>G

Posted Date: August 17th, 2021

DOl: https://doi.org/10.21203/rs.3.rs-779145/v1 
License: (c) (i) This work is licensed under a Creative Commons Attribution 4.0 International License. Read Full License 


\section{Abstract}

\section{Background}

Intellectual disability is a generalized neurodevelopmental disorder characterized by significantly impaired intellectual and adaptive functioning. About a quarter of cases are caused by genetic diseases, and about 5 percent are inherited from a person's parents. The objective of this study was to explore the candidate disease-causing gene in an intellectual disability family.

\section{Methods}

Whole genome sequencing was performed on affected twins, their affected children, and unaffected parents, and data filtration for most rare variants and In Silico prediction tools to determine the pathogenecity. Further, Sanger sequencing was used to validate the causative mutation.

Results

In the family, a previously undescribed heterozygous variant in $Z B T B 18$ gene c.1323C $>\mathrm{G}$ (p.His441GIn) was identified. The mutation co-segregated with all affected individuals in the family and was not found in unaffected members.

Conclusions

c.1323C $>$ G mutation in ZBTB18 gene on 1 chromosome may be related with the phenotype of intellectual disability in this family. WGS is an efficient method to perform molecular diagnosis for hereditary intellectual disability.

\section{Background}

Intellectual disability (ID) is a generalized neurodevelopmental disorder characterized by substantial impairment in intellectual functioning (reasoning, learning, problem solving) and adaptive behaviour (conceptual, social and practical skills) that originate in the developmental period, and affects $1 \%$ to $3 \%$ children [1, 2]. ID can occur in isolation or in combination with congenital malformations, other neurological features such as epilepsy, sensory impairment and autism spectrum disorders (ASD), and its severity (mild, moderate, severe and profound) is highly variable [3, 4].ID pose medical, financial, and psychological challenges on individuals, families, health-care systems, and societies [5].

ID is genetically and phenotypically extremely heterogeneous [3]. There are many etiologies of ID: genetic causes (eg, chromosomal abnormalities, copy number variants (CNVs), or mutations), environmental causes (eg, alcohol and other teratogens, prenatal infections), traumatic brain injury, neurologic/brain disorders, nutritional deficiencies, and inborn errors of metabolism $[3,6]$. Among them, genetic factors are regarded as one of the most prominent etiologies. 
Many studies have been performed to reveal the genetic etiology of ID. The traditional testing methods for ID diagnosis include karyotype, microarray, polymerase chain reaction, Fragile $\mathrm{X}$, fluorescent in situ hybridization and mitochondrial DNA testing [7]. Chromosomal microarray analysis (CMA), comparative genomic hybridization (CGH) or SNP arrays, has long been the first-tier test for the child with ID of unknown etiology [2,8]. Many previous studies on the genetic etiologies of ID were based on CMA [911]. These methods have intrinsic specific limitations. Karyotype and microarray can detect the whole genome of an individual's with low resolution, while the latter (polymerase chain reaction, Fragile X, fluorescent in situ hybridization, and mitochondrial DNA testing) has higher resolution with only a small part of a person's genome.

In recent years, with the rapid development of next generation sequencing (NGS), as well as NGS approaches including whole-exome sequencing (WES) and whole-genome sequencing (WGS) can provide both broad and high-resolution identification of genetic variants. WES and WGS are more and more widely used to identify pathogenic genes for ID [12-21]. According to the results of previous studies on X-linked, autosomal-dominant and autosomal-recessive ID, over 700 genes and 130 rare CNVs have been identified, which can be used for the genetic diagnosis of both ID and ID-associated disorders [3, 22]. In the current study, we performed whole genome sequencing in an ID family to identify the candidate gene.

\section{Methods}

\section{Subjects Clinical samples}

An ID family with three-generation-span from Sichuan province in China was recruited after informed consent (Figure 1). Two affected (III:1, III:2) members received full clinical evaluation. The blood samples of four affected冈II:2, II:3, III:1, III:2『and two healthy members (I:1,I:2) were collected for further studies. Clinical records and radiographic images were published under the patients' written permission. The study was approved by the Ethics Committee of West China Second University Hospital, Sichuan University (No: 2015011) and adhered to the tenets of the Declaration of Helsinki.

\section{DNA extraction and whole genome sequencing}

According to manufacturer's instructions, Genomic DNA was isolated from peripheral blood leukocytes, collected from participants using DNA QIAamp mini kit (Qiagen, Hilden, Germany). WGS was performed on 4 patients (II:2,Il:3,III:1,III:2) and 2 normal family members (I:1,l:2), were sequenced by BGI-seq 500 with $50 \mathrm{bp}$ paired-end.

\section{Bioinformatics analysis}

We used SOAP nuke to remove the adapters and low-quality reads, then the reads were mapped to the human genome reference (UCSC GRCh37/hg19) by Burrows-Wheeler aligner (BWA-MEM, version 0.7.10) [23]. Variants calling was performed using the Genome Analysis Tool Kit (GATK, version 3.3) [24]. Variant Effect Predictor (VEP) was used to annotate and classify all the variants [25]. Then we screened variants 
based on their frequency in the public and internal databases (e.g, 1000 genome, GnomAD, and our internal database), and we only retained variants with a Minor Allele Frequency (MAF) $<0.005$. After that we do a filtration depending on the inheritance model of the pedigree. At last we do the prediction for harmful candidate variants using some software including Sift (http://www.sift.jcvi.org), PolyPhen2 (http://www. genetics.bwh.harvard.edu/pph2/).

\section{Sanger validation}

We designed all polymerase chain reaction primers for validation by Primer 5.0. The candidate variants were verified by Sanger sequencing to filter out false positive variants. The six family members (affected individuals II:2,II:3,III:1,IIl:2; unaffected individuals I:1,l:2) were sequenced by bidirectional Sanger sequencing to determine co-segregation of the candidate mutations. Polymerase chain reaction (PCR) and sequencing primers are available upon request.

\section{Results}

\section{Clinical features}

Four individuals in this family have mild ID. The proband (III:1), was a 7-year-old male patient. At the age of 31 months, Magnetic resonance (MR) scan of the brain and language impairment screening assessment were performed. The MR result revealed dysplasia of corpus callosum. The process of language development were as follows: The overall language ability was at the level of 11-12 months old. His speech related ability was at the level of a 9 months old. His ability of auditory related expression was at the level of a 12-13 months old. His ability of visual expression was at the level of a 12-13 months old. At the age of 6 years, the Magnetic resonance imaging (MRI) examination showed that the posterior horn of both ventricles was enlarged, the corpus callosum was changed, and the hippocampus was small. Electroencephalogram (EEG) examination suggested abnormality.

As for patient III:2『at the age of 19 months $\llbracket$ general examination was performed. MR scan of the brain indicated mild paraventricular white matter softening. The Intelligence Development Diagnostic Scale showed that the total DQ of the children was 47.2, and the overall level of intelligence development was significantly lower than that of the normal children of the same age. Among them, the ability to cope with

people was equivalent to 9 months old, the ability to cope with things was equivalent to 8 months old, the ability of gross motor was equivalent to 12 months old, the ability of fine motor was equivalent to 8 months old and the ability of speech was equivalent to 8 months old. III:1 and III:2 are currently attending special schools for the ID.

As for patients II:2 and ,I:3, there were no clinical diagnostic data. According to their parents' description, they are identical twins and often had a fever after weaning at 1 years of age. Once fever occurred, there would be convulsions. After 3 or 4 times, the convulsion occurred when the body temperature was only $37.5^{\circ} \mathrm{C}$. The doctor diagnosed as possible epilepsy. After taking the medicine, the incidence of epilepsy was less. Under the guidance, they can only do ordinary housework, such as 
cooking, washing dishes, sweeping the floor and so on. But they can't go shopping alone. If they pay more and the salesman doesn't return the extra money to them, they don't know what to ask for.

\section{Mutation detection}

To identify the causative variants in the ID family, we performed WGS described in the methods. Highquality results were obtained, with mean coverage in excess of $91 \%$, and the average depth $>40 \mathrm{X}$ (Table 1).

Table 1 Average depth and coverage of WGS

\begin{tabular}{|lllll|}
\hline Sample & Q20 & Q30 & Average depth & Coverage (\%) \\
\hline $\mathrm{I}: 1$ & 98.05 & 90.90 & 44.41 & 92.35 \\
\hline $\mathrm{I}: 2$ & 98.36 & 92.17 & 45.28 & 91.83 \\
$\mathrm{II}: 2$ & 98.40 & 92.05 & 45.72 & 91.81 \\
\hline $\mathrm{II}: 3$ & 98.44 & 92.19 & 43.09 & 91.82 \\
\hline $\mathrm{III}: 1$ & 98.47 & 92.42 & 43.97 & 92.35 \\
\hline $\mathrm{III}: 2$ & 98.00 & 90.65 & 45.27 & 91.81 \\
\hline
\end{tabular}

Following bioinformatics analysis, a de novo mutation c.1323C $>\mathrm{G}$, which was described in reference to RefSeq transcript NM_205768.2, was found in Exon2 of ZBTB18 gene completely segregated in the affected family members. The mutation causes an amino acid change from histidine to glutamine at position 441 (p.His441GIn). This variant is predicted to be probably damaging (Polyphen score 0.997) and deleterious (SIFT score 0 ).

Following this result, we applied a further mutation validation strategy, by Sanger sequencing, with family members. Sanger sequencing was performed with specific primers, confirmed the co-segregation of the mutation (Figure 2). Multiple orthologous sequence alignment revealed that 441 codon alanine of ZBTB18 was highly conserved amino acids across different species (Figure 3 ). This indicates that any mutation at those codons may have a deleterious effect. This variant has not been reported in HGMD database or ClinVar database.

\section{Discussion}

In the present study, we performed whole genome sequencing in an ID family. After systematic NGS data analysis and Sanger sequencing verification, we identified a new heterozygous missense mutation (c.1323C>G, p.His441GIn) in ZBTB18(NM_205768.2) in the four patients except the two unaffected individuals of the family. 
ZBTB18 gene, previously known as ZNF238 or RP58, encodes a transcriptional repressor of BTB (broad complex tramtrack bric-a-brac) zinc finger family, which is composed of an N-terminal BTB domain mediating protein-protein interaction and four Cys2-His2-like $(\mathrm{C} 2 \mathrm{H} 2)$ zinc fingers mediating proteinbinding-to- regulator within promoters at its $\mathrm{C}$-terminus respectively. BTB domain zinc finger factors linked to development of the mammalian cerebellum, cerebral cortex and macroglia [26, 27]. ZBTB18 is activated during neuronal differentiation in pin-like cells of the ventricular zone, and in migrating multipolar cells [28]. ZBTB18 participates in neuron and astrocyte differentiation by mediating cell-cycle control of neural stem cells [29]. ZBTB18 is essential for the growth and organization of the cerebellum and regulates the development of both GABAergic and glutamatergic neurons [30]. ZBTB18 acts to restrict the represses expression of pax6, ngn2 and neuroD1, and expression of these three sequential proneurogenic genes causes intermediate neurogenic progenitors (INP) to differentiate and migrate. Importance of time- and place-specific expression is hypothesized [31]. ZBTB18 mediates multipolar-tobipolar transition of migrating cortical neurons by inhibiting the transcription factors Ngn2and Rnd2 [32, 33].

ZBTB18 gene is extremely intolerant to genetic variation [34]. In humans, genetic mutations in ZBTB18 are associated with structural brain abnormalities, neuronal migration disorder and ID $[12,14,16,17,35-$ 48]. These mutations are located in the BTB domain, $\mathrm{C} 2 \mathrm{H} 2$ zinc finger domain or other sites of ZBTB18 protein. The types of mutations include missense, nonsense, small deletion, small insertion, and gross deletion. Nonsense, small deletion and small insertion lead to truncated ZBTB18 protein with a heterozygous loss of the zinc finger domain, this could be considered haploinsufficiency. Gross deletion lead to the total loss of ZBTB18 protein. The researchers suggest that haploinsufficiency/loss-of-function represents a general pathological mechanism for disease $[40,46]$. Most of the reported missense variants are clustered in the second, third and fourth zinc finger domain. Variants in a zinc finger domain could affect DNA-binding properties, impaired binding of ZBTB18 to DNA will disturb its function as transcriptional repressor $[41,46]$. In addition, mutating the zinc finger could have a dominant-negative effect by rendering the wild-type protein unable to bind DNA because it dimerizes with a mutant protein [41]. It has been suggested that altered transcriptional regulation could represent an important pathological mechanism for ZBTB18 missense variants in human disease [49].

The novel missense mutation c.1323C>G (p.His441GIn) of ZBTB18gene was

predicted to be pathogenic based on supportive information. First, it is absent from the normal population and National Center for Biotechnology Information (NCBI) single nucleotide polymorphism database. Second, the mutation loci is highly conserved amino acids across different species, and the alteration it produces in the chemical properties of amino acids is significant. Functional studies are further required to confirm the potential effect of the mutation on the protein.

Identifying the disease-causing mutation in patients with ID is necessary to provide proper genetic counseling and guiding gene-specific therapies in the future. The extensive genetic heterogeneity of this disorder requires a genome-wide detection of all types of genetic variation. WGS enabled sequencing of 
the entire 3 billion bases of the human genome, including both coding and noncoding DNA. In recent years, the cost of WGS has gradually decreased. Therefore, WGS is increasingly being used to search causative mutations in order to get an accurate molecular diagnosis $[13,18,19,21]$. Our study also shows that WGS is an efficient method to perform molecular diagnosis for hereditary ID.

In conclusion, the c.1323C>G (p.His441GIn) mutation of ZBTB18 gene expands the spectrum of mutations that causes ID. This study provide supporting evidence that WGS is a highly efficient strategy to provide a molecular diagnosis for ID.

\section{Abbreviations}

ID: intellectual disability; CNVs: copy number variants; CMA: chromosomal microarray analysis; NGS: next generation sequencing; WES: whole-exome sequencing; WGS: whole-genome sequencing; MR: magnetic resonance; MRI: magnetic resonance imaging.

\section{Declarations}

\section{Ethics approval and consent to participate}

The study was approved by the Ethics Committee of West China Second University Hospital, Sichuan University (No: 2015011) and adhered to the tenets of the Declaration of Helsinki.

\section{Consent for publication}

Not applicable.

\section{Availability of data and materials}

All data are contained in the article.

\section{Competing interests}

The authors declare that they have no conficts of interest with the contents of this article. Funding

This study was supported by the National Key Research and Development Program of China (No.2016YFC1000102, 2018YFC1002203), and the Program for Changjiang Scholars and Innovative Research Team in University (ID: IRT0935).

\section{Authors' contributions}

Nana Li and Ping Yu developed the study design. Nana Li, Lu Li, Meixian Wang and Ping Yu conducted the experiment and drafted the manuscript. Yanna Zou, Zhen Liu, Ying Deng and Hong Kang assisted in analyzing the data. Yanna Zou and Hong Qin participated in the clinical evaluation of the patients. 
Yanping Wang and Jun Zhu participated in reviewing the manuscript. All authors have read and approved the final manuscript.

\section{Acknowledgements}

We are very grateful to individuals in the family for their kind agreement to participate in this study.

\section{Authors' information}

${ }^{1}$ National Center for Birth Defect Monitoring, West China Second University Hospital, Sichuan University, Chengdu, Sichuan, China. ${ }^{2}$ Key Laboratory of Birth Defects and Related Diseases of Women and Children (Sichuan University), Ministry of Education, Chengdu, Sichuan, China. ${ }^{3}$ Department of gynaecology and obstetrics, Changyi Maternal and Child care Hospital, Changyi, Shandong, China. ${ }^{4}$ Department of gynaecology and obstetrics, wuhou district people's hospital, Chengdu, Sichuan, China.

\section{References}

1. Salvador-Carulla L, Reed GM, Vaez-Azizi LM, Cooper SA, Martinez-Leal R, Bertelli M, et al. Intellectual developmental disorders: towards a new name, definition and framework for "mental retardation/intellectual disability" in ICD-11. World Psychiatry. 2011; 10:175-80.

2. Moeschler JB, Shevell M. Comprehensive evaluation of the child with intellectual disability or global developmental delays. Pediatrics. 2014; 134:e903-18.

3. Vissers LE, Gilissen, C, Veltman, JA. Genetic studies in intellectual disability and related disorders. Nat Rev Genet. 2016;17: 9-18.

4. Vasudevan P, Suri, M. A clinical approach to developmental delay and intellectual disability. Clin Med (Lond). 2017;17: 558-61.

5. Feldkamp ML, Carey JC, Byrne JLB, Krikov S, Botto LD. Etiology and clinical presentation of birth defects: population based study. BMJ. 2017; 357: j2249.

6. Purugganan, O. Intellectual Disabilities. Pediatr Rev. 39, 299-309 (2018).

7. Sun F, Oristaglio J, Levy SE, Hakonarson H, Sullivan N, Fontanarosa J, et al. Genetic Testing for Developmental Disabilities, Intellectual Disability, and Autism Spectrum Disorder. Agency for Healthcare Research and Quality (US). 2015.

8. $\quad$ Li Y, Anderson LA, Ginns El, Devlin JJ. Mol Diagn Ther. 2018; 22: 129-38.

9. Chong WW, Lo IF, Lam ST, Wang CC, Luk HM, Leung TY, et al. Performance of chromosomal microarray for patients with intellectual disabilities/developmental delay, autism, and multiple congenital anomalies in a Chinese cohort. Mol Cytogenet. 2014; 7: 34. 
10. Capobianco S, Lava SA, Bianchetti MG, Martinet D, Belfiore M, Ramelli GP, et al. Chromosomal microarray among children with intellectual disability: a useful diagnostic tool for the clinical geneticist. Dev Med Child Neurol. 2014; 56: 290.

11. Fan $Y$, Wu Y, Wang L, Wang Y, Gong Z, Qiu W, et al. Chromosomal microarray analysis in developmental delay and intellectual disability with comorbid conditions. BMC Med Genomics. 2018, 11: 49.

12. Rauch A, Wieczorek D, Graf E, Wieland T, Endele S, Schwarzmayr T, et al. Range of genetic mutations associated with severe non-syndromic sporadic intellectual disability: an exome sequencing study. Lancet. 2012; 380:1674-82.

13. Gilissen C, Hehir-Kwa JY, Thung DT, van de Vorst M, van Bon BW, Willemsen MH, et al. Genome sequencing identifies major causes of severe intellectual disability. Nature. 2014; 511: 344-7.

14. Srivastava S, Cohen JS, Vernon H, Baranano K, McClellan R, Jamal L, et al. Clinical whole exome sequencing in child neurology practice. Ann Neurol. 2014; 76: 473-83.

15. Farwell Hagman KD, Shinde DN, Mroske C, Smith E, Radtke K, Shahmirzadi L, et al. Candidate-gene criteria for clinical reporting: diagnostic exome sequencing identifies altered candidate genes among $8 \%$ of patients with undiagnosed diseases. Genet Med. 2016; 19: 224-35.

16. Bowling KM, Thompson ML, Amaral MD, Finnila CR, Hiatt SM, Engel KL, et al. Genomic diagnosis for children with intellectual disability and/or developmental delay. Genome Med. 2017; 9: 43.

17. Popp B, Ekici AB, Thiel CT, Hoyer J, Wiesener A, Kraus C, et al. Exome Pool-Seq in neurodevelopmental disorders. Eur J Hum Genet. 2017; 25: 1364-76.

18. Lindstrand A, Eisfeldt J, Pettersson M, Carvalho CMB., Kvarnung M, Grigelioniene G, et al. From cytogenetics to cytogenomics: whole-genome sequencing as a first-line test comprehensively captures the diverse spectrum of disease-causing genetic variation underlying intellectual disability. Genome Med. 2019; 11: 68.

19. Thuresson AC, Soussi Zander C, Zhao JJ, Halvardson J, Maqbool K, Mansson E, et al. Whole genome sequencing of consanguineous families reveals novel pathogenic variants in intellectual disability. Clin Genet. 2019; 95: 436-9.

20. Han JY, Lee IG. Genetic tests by next-generation sequencing in children with developmental delay and/or intellectual disability. Clin Exp Pediatr. 2020, 63: 195-202.

21. Wang J, Wang Y, Wang L, Chen WY, Sheng M. The diagnostic yield of intellectual disability: combined whole genome low-coverage sequencing and medical exome sequencing. BMC Med Genomics. 2020; $13: 70$. 
22. Kochinke K, Zweier C, Nijhof B, Fenckova M, Cizek P, Honti F, et al. Systematic Phenomics Analysis Deconvolutes Genes Mutated in Intellectual Disability into Biologically Coherent Modules. Am J Hum Genet. 2016; 98: 149-64.

23. Vasimuddin Md SM, Heng L, Srinivas A. Efficient Architecture-Aware Acceleration of BWA-MEM for Multicore Systems. IEEE International Parallel and Distributed Processing Symposium (IPDPS), Rio de Janeiro, Brazil. 2019. p. 314-324.

24. DePristo MA, Banks E, Poplin R, Garimella KV, Maguire JR, Hartl C, et al. A framework for variation discovery and genotyping using next-generation DNA sequencing data. Nat Genet. 2011; 43: 491-8.

25. McLaren W, Gil L, Hunt SE, Riat HS, Ritchie GR, Thormann A, et al. The Ensembl Variant Effect Predictor. Genome Biol. 2016; 17: 122.

26. Aoki K, Meng G, Suzuki K, Takashi T, Kameoka Y, Nakahara K, et al. RP58 associates with condensed chromatin and mediates a sequence-specific transcriptional repression. J Biol Chem. 1998; 273: 26698-704.

27. Mitchelmore C, Kjaerulff KM, Pedersen HC, Nielsen JV, Rasmussen TE, Fisker MF, et al. Characterization of two novel nuclear BTB/POZ domain zinc finger isoforms. Association with differentiation of hippocampal neurons, cerebellar granule cells, and macroglia. J Biol Chem. 2002; 277: 7598-609.

28. Ohtaka-Maruyama C, Hirai S, Miwa A, Takahashi A, Okado H. The 5'-flanking region of the RP58 coding sequence shows prominent promoter activity in multipolar cells in the subventricular zone during corticogenesis. Neuroscience. 2012; 201: 67-84.

29. Hirai S, Miwa A, Ohtaka-Maruyama C, Kasai M, Okabe S, Hata Y, et al. RP58 controls neuron and astrocyte differentiation by downregulating the expression of Id1-4 genes in the developing cortex. EMBO J. 2012; 31: 1190-202.

30. Baubet V, Xiang C, Molczan A, Roccograndi L, Melamed S, Dahmane N. Rp58 is essential for the growth and patterning of the cerebellum and for glutamatergic and GABAergic neuron development. Development. 2012; 139: 1903-9.

31. Xiang C, Baubet V, Pal S, Holderbaum L, Tatard V, Jiang P, et al. RP58/ZNF238 directly modulates proneurogenic gene levels and is required for neuronal differentiation and brain expansion. Cell Death Differ. 2012; 19: 692-702.

32. Ohtaka-Maruyama C, Hirai S, Miwa A, Heng Jl, Shitara H, Ishii R, et al. RP58 regulates the multipolar-bipolar transition of newborn neurons in the developing cerebral cortex. Cell Rep. 2013; 3: 45871. 
33. Heng Jl, Qu Z, Ohtaka-Maruyama C, Okado H, Kasai M, Castro D, et al. The zinc finger transcription factor RP58 negatively regulates Rnd2 for the control of neuronal migration during cerebral cortical development. Cereb Cortex. 2015; 25: 806-16.

34. Lek M, Karczewski KJ, Minike, EV, Samocha KE, Banks E, Fennell T, et al. Analysis of protein-coding genetic variation in 60,706 humans. Nature. 2016; 536: 285-91.

35. Nagamani SC, Erez A, Bay C, Pettigrew A, Lalani SR, Herman K, et al. Delineation of a deletion region critical for corpus callosal abnormalities in chromosome 1q43-q44. Eur J Hum Genet. 2012; 20:176-9.

36. Ballif BC, Rosenfeld JA, Traylor R, Theisen A, Bader PI, Ladda RL, et al. High-resolution array CGH defines critical regions and candidate genes for microcephaly, abnormalities of the corpus callosum, and seizure phenotypes in patients with microdeletions of 1q43q44. Hum Genet. 2012; 131:145-56.

37. Perlman SJ, Kulkarni S, Manwaring L, Shinawi M. Haploinsufficiency of ZNF238 is associated with corpus callosum abnormalities in 1q44 deletions. Am J Med Genet A. 2013; 161A: 711-6.

38. de Munnik SA, Garcia-Minaur S, Hoischen A, van Bon BW, Boycott KM, Schoots J, et al. A de novo non-sense mutation in ZBTB18 in a patient with features of the 1q43q44 microdeletion syndrome. Eur $\mathrm{J}$ Hum Genet. 2014; 22: 844-6.

39. Lopes F, Barbosa M, Ameur A, Soares G, de Sa J, Dias Al, et al. Identification of novel genetic causes of Rett syndrome-like phenotypes. J Med Genet. 2016; 53: 190-9.

40. Depienne C, Nava C, Keren B, Heide S, Rastetter A, Passemard S, et al. Genetic and phenotypic dissection of 1q43q44 microdeletion syndrome and neurodevelopmental phenotypes associated with mutations in ZBTB18 and HNRNPU. Hum Genet. 2017; 136: 463-79.

41. Cohen JS, Srivastava S, Farwell Hagman KD, Shinde DN, Huether R, Darcy D, et al. Further evidence that de novo missense and truncating variants in ZBTB18 cause intellectual disability with variable features. Clin Genet. 2017; 91: 697-707.

42. Prevalence and architecture of de novo mutations in developmental disorders. Nature. 2017; 542: 433-8.

43. Ehmke N, Karge S, Buchmann J, Korinth D, Horn D, Reis O, et al. A de novo nonsense mutation in ZBTB18 plus a de novo 15q13.3 microdeletion in a 6-year-old female. Am J Med Genet A. 2017; 173: 1251-6.

44. Evers C, Staufner C, Granzow M, Paramasivam N, Hinderhofer K, Kaufmann L, et al. Impact of clinical exomes in neurodevelopmental and neurometabolic disorders. Mol Genet Metab. 2017; 121: 297307. 
45. Kosmicki JA, Samocha KE, Howrigan DP, Sanders SJ, Slowikowski K, Lek M, et al. Refining the role of de novo protein-truncating variants in neurodevelopmental disorders by using population reference samples. Nat Genet. 2017; 49: 504-10.

46. van der Schoot V, de Munnik S, Venselaar H, Elting M, Mancini GMS, Ravenswaaij-Arts CMA, et al. Toward clinical and molecular understanding of pathogenic variants in the ZBTB18 gene. Mol Genet Genomic Med. 2018; 6: 393-400.

47. Trinh J, Kandaswamy KK, Werber M, Weiss MER, Oprea G, Kishore S, et al. Novel pathogenic variants and multiple molecular diagnoses in neurodevelopmental disorders. J Neurodev Disord. 2019; 11: 11 .

48. Vetrini F, McKee S, Rosenfeld JA, Suri M, Lewis AM, Nugent KM, et al. De novo and inherited TCF20 pathogenic variants are associated with intellectual disability, dysmorphic features, hypotonia, and neurological impairments with similarities to Smith-Magenis syndrome. Genome Med. 2019; 11: 12.

49. Hemming IA, Clement O, Gladwyn-Ng IE, Cullen HD, Ng HL, See HB, et al. Disease-associated missense variants in ZBTB18 disrupt DNA binding and impair the development of neurons within the embryonic cerebral cortex. Hum Mutat. 2019; 40: 1841-55.

\section{Figures}

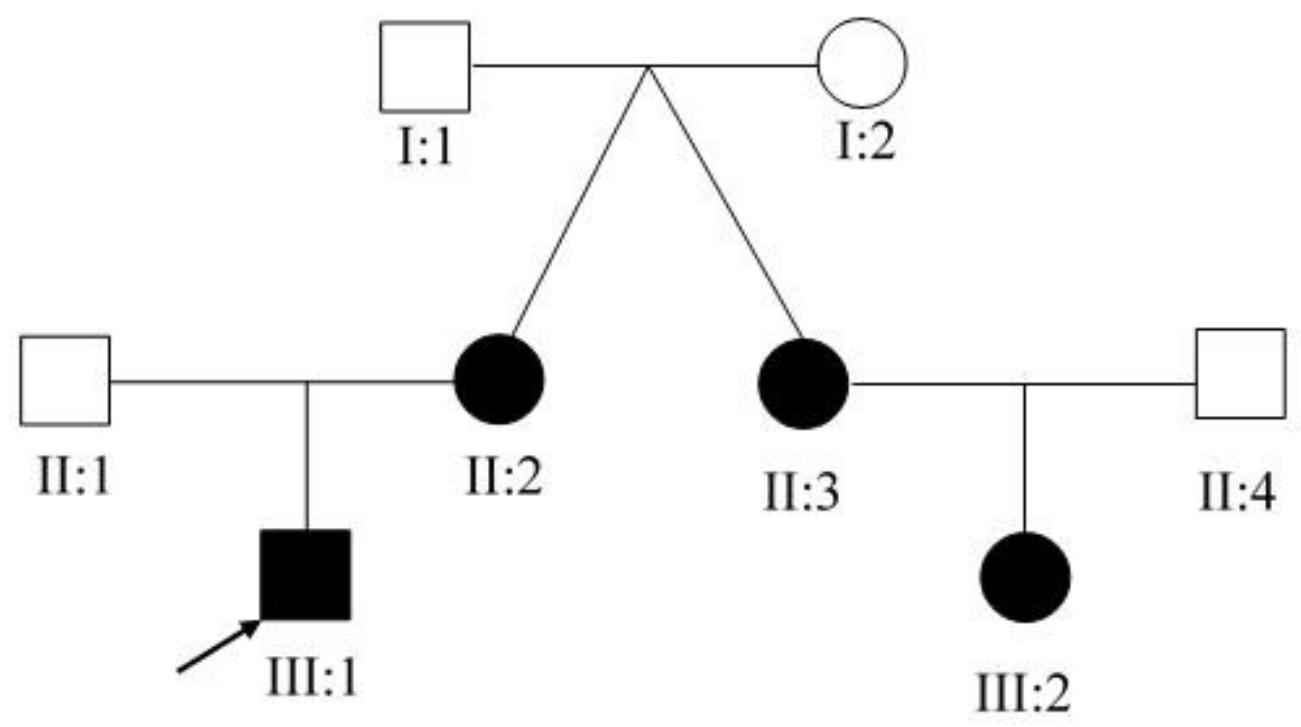

Figure 1

Pedigree of the ID family 
c. $1323 \mathrm{CG}$

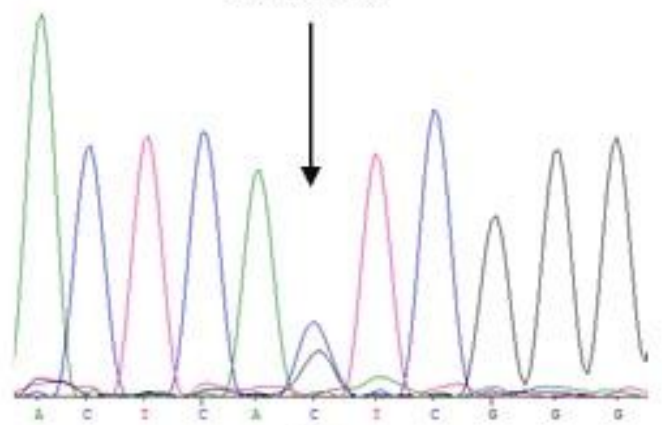

II:1

c. $1323 \mathrm{CG}$

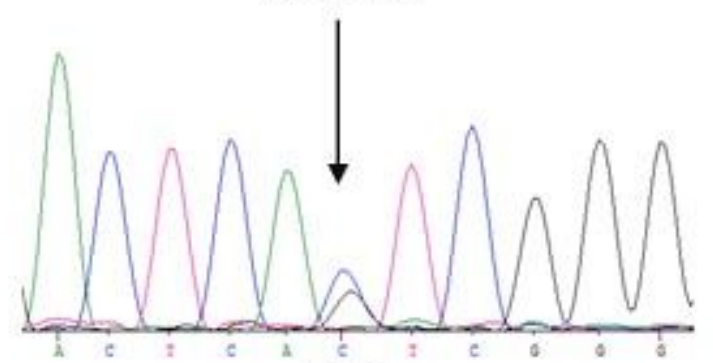

III: 1

c. $1323 \mathrm{CC}$

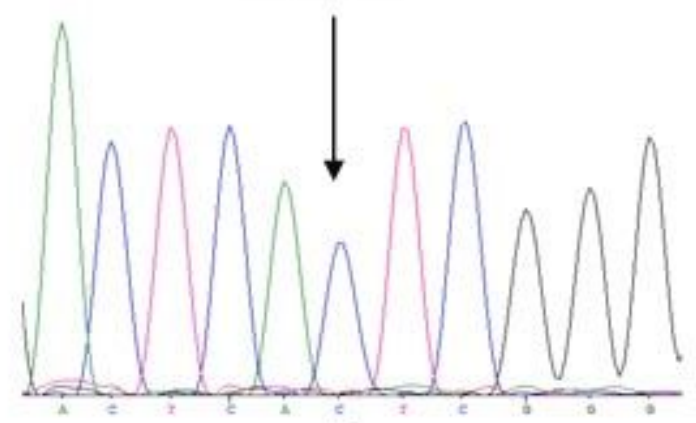

$\mathrm{I}: 1$ c. $1323 \mathrm{CG}$

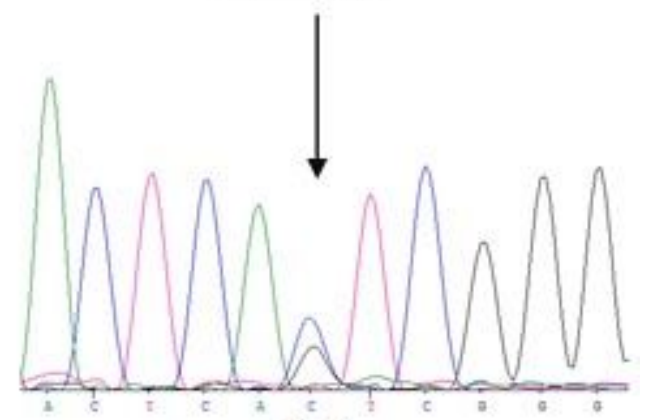

II: 2

c. $1323 \mathrm{CG}$

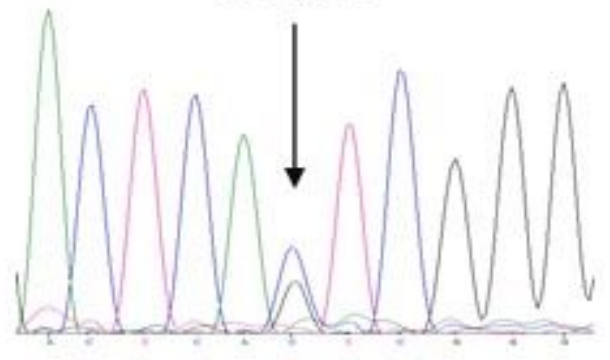

III:2

c. $1323 \mathrm{CC}$

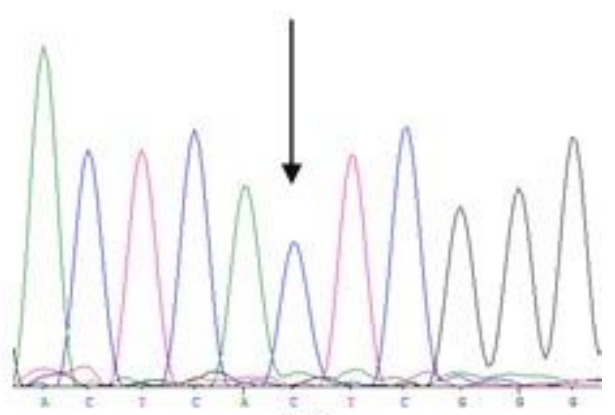

$\mathrm{I}: 2$

\section{Figure 2}

The validation results of Sanger sequencing of ZBTB18 ((NM_205768)

Chimpanzee-ZBTB18

Giant_panda-ZBTB18

Human-ZBTB18

Mouse-Zbtb18

Xen_laevis-zbtb18

Xen_tropicalis-zbtb18

Zebrafish-zbtb18
FSCIYTLKRHERT F GEKPYTCTQCGKSFQYSHNLSRHAVVHTREKPHAC FSCIYTLKRHERT FGEKPYTCTQCGKSFQYSHNLSRHAVVHTREKPHAC FSCMYTLKRHERT 1 GGEKPYTCTQCGKSFQYSHNLSRHAVVHTREKPHAC FSCIYTLKRHERT| GGEKPYTCTQCGKSFQYSHNLSRHAVVHTREKPHAC FSCMYTLKRHERT PGEKPYTCTQCGKSFQYSHNLSRHAVVHTREKPHAC FSCIYTLKRHERT FGEKPFTCTQCGKSFQYSHNLSRHAVVHTREKPHAC FSCIYTLKRHERT 1 GGEKPYTCTTCGKSFQYSHNLSRHAVVHTREKPHAC

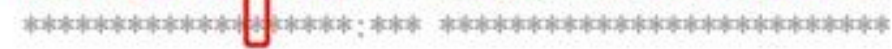

\section{Figure 3}

Alignment of ZBTB18 protein sequences across species 